\title{
El contrato psicológico y su relación con el compromiso organizacional
}

\section{The psychological contract and its relation with the organizational commitment}

María Auxiliadora Guerrero Bejarano

Universidad Internacional del Ecuador, Ecuador

Autor para correspondencia: maguerrerobe@uide.edu.ec

Fecha de recepción: 12 de Septiembre de 2016 - Fecha de aceptación: 01 de Noviembre de 2016

\section{Resumen}

El compromiso organizacional es comúnmente definido como un enlace psicológico entre el empleado y la organización para la que trabaja (Kuo, 2013), este vínculo haría menos posible que el colaborador abandone voluntariamente su trabajo. El compromiso organizacional tendría relación con el comportamiento y actitudes del trabajador hacia la empresa, razón por la que es uno de los constructos más estudiados. Este articulo hara un estudio literario entre la relacion en el contrato psicológico y el compromiso organizacional.

Palabras claves: contrato psicológico; compromiso organizacional; liderazgo

\begin{abstract}
Organizational commitment is commonly defined as a psychological link between the employee and the organization for which he or she works (Kuo, 2013), this link would make it less likely that the employee voluntarily leaves his or her job. The organizational commitment would be related to the behavior and attitudes of the worker towards the company, which is why it is one of the most studied constructs. This article will make a literary study between the relationship in the psychological contract and the organizational commitment.
\end{abstract}

Key words: psychological contract; organizational commitment; leadership 


\section{Introducción}

Debido a los cambios actuales los entornos empresariales ha sufrido variaciones, las fuerzas de los mercados han obligado a las empresas a revisar la forma en la que manejan su recurso humano, estudios previos resaltan la necesidad económica cada vez mayor de prácticas de trabajo más flexibles (Millward \& Hopkins, 1998). Las organizaciones están aumentando su dependencia de la participación de los empleados, ya que su éxito depende de la capacidad de la empresa para aprovechar las habilidades y conocimientos de los colaboradores; buscando mantener el nivel de competitividad las empresas buscan fomentar la iniciativa de los empleados; estos climas proactivos requieren de mayores habilidades de liderazgo, por parte de los jefes o gerentes, que las utilizadas tradicionalmente para que puedan desarrollar en los colaboradores un sentido de compromiso y pertenencia.(Sethuraman \& Suresh, 2014). El liderazgo es definido como la habilidad de influir a un grupo para la consecución de metas, en el mundo actual, se necesitan líderes que tengan visión de futuro y puedan inspirar a los miembros de la organización a alcanzar estas visiones (Robbins, Judge, Millett, \& Boyle, 2013). Existen estudios que comprobarían que el estilo de liderazgo que un jefe o gerente aplica influiría en desempeño de los equipos de trabajo y por consiguiente de la organización (Dionne, Yammarino, Atwater, \& Spangler, 2004; Politis, 2002; Vigoda-Gadot, 2007).

Los estilos de liderazgo transformacional y transaccional no son opuestos, de acuerdo con Bass el liderazgo transformacional sería una expansión del liderazgo transaccional. (Bass, 1999) Se podría decir que el líder transformacional es un líder transaccional con carisma que influye a que sus seguidores vayan más allá de lo que formalmente se les demanda(Cardona, 2000) Un estudio previo demostró que parecería justo decir que para obtener buenos logros los líderes necesitarían de una combinación de los dos estilos (Bass, Avolio, Jung, \& Berson, 2003) Bass definió que el liderazgo transaccional asentaría las bases de las relaciones entre los líderes y los seguidores, estableciendo las reglas y especificando las expectativas que se tienen en la relación, aclarando responsabilidades, negociando contratos y las formas de recompensas y reconocimiento para conseguir los objetivos y rendimiento esperados, mientras el liderazgo transformacional promovería el desarrollo de los seguidores desafiándolos a pensar en formas nuevas e inspirándolos a ir más allá de lo que se espera de ellos, motivándolos a mantener los valores y normas morales que serían guía de su comportamiento (Bass, 1988). El liderazgo transformacional puede elevar los valores de los empleados y mejorar la eficiencia de la organización a través de la consideración individualizada, estimulación intelectual y la comunicación que los líderes tendrían con los miembros de sus equipos (Wang \& Howell, 2010; Yeh, 2008).

El cambio tecnológico y su constante evolución ha generado que las empresas busquen no solo reaccionar al cambio, sino promoverlo para mantener sus niveles de competitividad, estos cambios conllevarían la búsqueda de estructuras organizacionales más compenetradas, es decir que sus niveles superiores buscarían tener una mayor comprensión de los niveles operativos; esto implicaría la necesidad de delegar responsabilidades de manera efectiva, por lo que el líder deberá ser capaz de manejar una organización que le permita conseguir que sus seguidores puedan manejar mayores niveles de responsabilidad e incertidumbre. Los líderes entonces deberían operar como agentes de cambio, siendo líderes transformacionales. Este tipo de líderes ayudan a alinear a los empleados con los valores y normas de las organizaciones. 
(Avolio, Waldman, \& Yammarino, 1991) Los líderes transformacionales elevan los deseos de sus seguidores para conseguir sus metas y autodesarrollo, mientras se promueve el desarrollo de grupos de trabajo. Los autores han definido cuatro componentes básicos en este estilo de liderazgo: la influencia idealizada (carisma), la consideración individualizada, la estimulación intelectual y la inspiración; por sus nombres en inglés estos componentes son conocidos como las cuatro "I"s del liderazgo transaccional. (Bass \& Avolio, 1990)El carisma o influencia idealizada está presente en los líderes que tiene visión y claramente definido el sentido de la misión de la empresa, generalmente los líderes que cuentan con este componente obtienen el extra esfuerzo de sus seguidores lo que le ayudaría a conseguir mejores resultados. La consideración individual, hace al líder concentrarse y diagnosticar las necesidad y capacidades de sus seguidores, lo que le permitiría definir tareas y responsabilidades y al mismo tiempo conseguir el desarrollo personal y profesional de sus seguidores. La estimulación intelectual, significa que el líder permanentemente está retando a sus seguidores a buscar nuevas formas de solucionar problemas, de esta manera generaría innovación en su equipo de trabajo. Finalmente la inspiración, que busca incrementar los niveles de motivación, optimismo, entusiasmo hacia el trabajo, para obtener los objetivos y metas planteados. Los líderes transformacionales buscan minimizar errores de forma proactiva, mediante la realimentación continua. (Bass \& Avolio, 1990).

El liderazgo transaccional está basado en el intercambio de recompensas entre el líder y los miembros de su equipo. Los empleados realizan su labor y a cambio el líder o directivo les proporciona recompensas económicas u otro tipo de refuerzos. Este tipo de liderazgo, puede conseguir efectos positivos en la satisfacción y rendimiento de los empleados si se aplica correctamente. (Bass, 1988) Tradicionalmente, el liderazgo transaccional era el más comúnmente utilizado, considerado eficaz porque facilita la consecución de objetivos. Los líderes transaccionales definen y comunican las tareas que deben realizar sus seguidores, y las recompensas que se recibirían si los objetivos son alcanzados. Como resultado los empleados comprenden sus roles en el trabajo y las expectativas que sus jefes tienen sobre ellos. Como resultado los colaboradores se motivan y dirigen sus esfuerzos para alcanzar las expectativas de su desempeño esperado. (Avolio et al., 1991) las habilidades y comportamientos que caracterizan el liderazgo transaccional (gratificaciones, recompensas y/o gestión de los contingentes por excepción) resultarían sencillos y han sido analizados ampliamente en la literatura para comprender en contraposición la no presencia del liderazgo o el liderazgo laissez-faire.

El compromiso organizacional es comúnmente definido como un enlace psicológico entre el empleado y la organización para la que trabaja (Kuo, 2013), este vínculo haría menos posible que el colaborador abandone voluntariamente su trabajo. El compromiso organizacional tendría relación con el comportamiento y actitudes del trabajador hacia la empresa, razón por la que es uno de los constructos más estudiados. (Meyer, Stanley, Herscovitch, \& Topolnytsky, 2002). El compromiso organizacional es usualmente medido en tres dimensiones establecidas por Allen \& Meyer, estas son: (a) el compromiso afectivo, deseo de los empleados de permanecer dentro de la organización de manera voluntaria, se caracteriza por tener una asociación afectiva y emocional, el empleado apreciaría el hecho de ser miembro de la organización, (b) el compromiso de continuidad o compromiso instrumental, las expectativas de los empleados de seguir en la organización o salir de ella basados en sus logros o de cómo serían considerados, relacionado también con los costos de salida de la organización y es construido sobre una base de 
relaciones de valor de alternativas posibles (c) compromiso normativo, permanecer en el trabajo debido a el sentimiento de culpa por dejar la organización, el sentimiento de obligación de permanecer en la organización, construido a partir de normas y expectativas de los empleados sobre la empresa (Farahani, Taghadosi, \& Behboudi, 2011; Komari \& Djafar, 2013; Shurbagi, 2014) En un estudio previo se demostró que existiría una relación positiva entre el compromiso organizacional y la satisfacción laboral y que esta relación no sería afectada por el género o la edad de los colaboradores (Anari, 2012). En otro estudio previo se habría comprobado la relación positiva entre el compromiso organizacional y el desempeño de la empresa pudiendo asumir que las empresas con mayores niveles de compromiso organizacional en sus colaboradores, obtendrían mejores resultados. (Faisal \& Al-Esmael, 2014; Kuo, 2013; Tims, Bakker, \& Xanthopoulou, 2011).

El compromiso organizacional es considerado una actitud muy deseable, pero difícil de alcanzar para muchos contextos organizacionales, una investigación anterior, siguiere que el compromiso organizacional puede tener un impacto positivo en las empresas tales como la reducción de la rotación del personal, menor ausentismo y una mayor productividad de los empleados (Cohen, 2007; Jernigan III, Beggs, \& Kohut, 2002). Los jefes influencian a los colaboradores para cumplir con ciertas tareas, en algunas circunstancias ofrecen algún tipo de recompensa para obtener mejores resultados en el trabajo. Estas tácticas tendrían efectos en el nivel del compromiso de los empleados hacia la organización y su comportamiento en el trabajo. (Chiu \& Ng, 2015; Chong, 2012; Jain, 2015; Moon, Hur, Ko, Kim, \& Yoon, 2014; Shepherd \& Mathews, 2000; Zayas-Ortiz, Rosario, Marquez, \& Gruñeiro, 2015).

La relación de los estilos de liderazgo y el compromiso organizacional ha sido estudiada por los académicos varias veces, encontrando que existe una relación positiva entre estas dos variables (Chan \& Mak, 2014; Kuo, 2013), así también la influencia del estilo de liderazgo en la satisfacción laboral (Abbasi \& Mohammadi, 2013; Ali, Sidow, \& Guleid, 2013; Arzi \& Farahbod, 2014) estos estudios demuestran una relación positiva entre estas variables. La naturaleza de los negocios sugiere que un estilo de liderazgo efectivo y el compromiso de los empleados hacia la organización podrían permitir obtener mejores resultados, de acuerdo con Meyer y Allen y su modelo multidimensional del compromiso organizacional, una empresa se encontrará en mejor posición para anticipar o afrontar cambios cuando la organización cuenta con empleados que han moldeado sus comportamientos y actitudes a partir de su compromiso organizacional (Meyer \& Herscovitch, 2001) Se ha demostrado que el estilo de liderazgo transformacional tendría una relación positiva con el compromiso organizacional, y en este caso que la inteligencia emocional sería una variable moderadora en esta relación. (Farahani et al., 2011) También se han relacionado estas variables incluyendo la cultura organizacional como una variable mediadora, encontrando que existiría una relación positivamente mediadora de la cultura organizacional sobre la relación de las dos variables, se supondría que la cultura afectaría al estilo de liderazgo y este al compromiso organizacional. (Shim, Jo, \& Hoover, 2015)

Con respecto a la relación del estilo de liderazgo con el compromiso organizacional existe un estudio previo identificado que analiza la relación entre el liderazgo transformacional y la cultura organizacional y el posible efecto moderador que podría existir en esta relación de la inteligencia emocional (Farahani et al., 2011). Rowden encontró, en un estudio previo, que es necesario que el líder se encuentre articulado con la visión, valores de la organización y con esa 
orientación ejercer su liderazgo, pensando en las necesidades de sus seguidores para conseguir mejores niveles de compromiso organizacional, en este estudio la edad fue analizada también y se encontró que las personas mayores tienden a estar más comprometidas con la organización debido a que tendrían menores opciones de otros empleos por lo estarían más comprometidos que los miembros más jóvenes de la organización. (Rowden, 2000) Los seguidores tendrían mayores niveles de compromiso hacia la empresa cuando sus líderes les brindan apoyo para el desarrollo de sus tareas y consecución de los objetivos. (Chan \& Mak, 2014; Joo, Yoon, \& Jeung, 2012). Farahani realizó un estudio en empresas de seguridad social en Irán, dónde analizó la relación del liderazgo transformacional sobre el compromiso organizacional, con la inteligencia emocional como variable mediadora, encontró que aunque existe una relación positiva entre las variables, la efectividad del liderazgo transformacional dependería del nivel de inteligencia emocional de los seguidores (Farahani et al., 2011).

El contrato psicológico para el trabajador está basado en sus percepciones que surgen como consecuencia de su relación laboral con la empresa, lo que significaría que este contrato sentaría las bases de dicha relación y sería predictor del compromiso organizacional, así como del comportamiento general de los colaboradores dentro de la organización (Solanes Puchol \& Martínez, 2012).

El concepto de contrato psicológico es vital para un análisis de los cambios en la naturaleza de la relación laboral, siendo un acuerdo no escrito que existe entre el individuo y la organización que se desarrolla junto con las condiciones de empleo (Millward \& Hopkins, 1998), este contrato señala temas de intercambio y de expectativas mutuas en la relación entre los colaboradores y las organizaciones para las que trabajan (Rousseau, 1985). La creencia de la existencia de obligaciones recíprocas suelen darse a partir de promesas explícitas, por ejemplo sistemas de recompensas (Rousseau, 1995). Cuanto más estable y consistentes son las peticiones y promesas de la organización, los empleados desarrollan una percepción inequívocas y uniformes de sus obligaciones así como de sus derechos (Rousseau, 1985). Los contratos pueden ser descritos en una línea continua que vas desde transaccional a relacional, se ha propuesto que la obligación transaccional está vinculado con el intercambio económico, mientras que las obligaciones relacionales están vinculadas con el intercambio social, a diferencia de los intercambios económicos, el intercambio social implica obligaciones no especificadas, cuyo cumplimiento depende de la confianza (Rousseau, 1989).

Actualmente, debido a los cambios producidos por la crisis económica, se puede observar grandes recortes de personal que ocurren con mayor frecuencia, por lo que se vuelve cada vez más importante mantener niveles apropiados de compromiso con los colaboradores así como contratos psicológicos con un contenido y estado adecuado, lo que generará ambientes y climas laborales estables y de mayor confianza; dónde la toma de decisiones de la alta gerencia podrían ser apreciadas como justas (Solanes Puchol \& Martínez, 2012).

\section{Conclusión}

De acuerdo con los estudios revisados, se podría concluir que los contratos psicológicos son importantes debido a su impacto en el desempeño de los empleados lo que se reflejaría en la calidad de los servicios entregados o en el nivel de la productividad de los mismos, por lo que las 
diferentes maneras en las que las empresas se desarrollan en su entorno y se relacionan con sus clientes serían consecuencia de la existencia de los contratos psicológicos dentro de la organización y su influencia en el nivel de compromiso organizacional de los empleados. Los trabajadores con contratos indefinidos mostrarían mayor compromiso organizacional y menor inseguridad laboral percibida que los trabajadores temporales, así mismo mayor contenido y cumplimiento así como mejor estado del contrato psicológico. Otros estudios también analizaron factores como la justicia percibida y la confianza depositada en la organización como factores que influyen el estado del contrato psicológico, ambos factores serían directamente influenciados por el estilo de liderazgo. (Millward \& Hopkins, 1998; Rosario-Hernández \& Millán, 2011; Silla, Gracia, Ferreira, \& Silla, 2006; Solanes Puchol \& Martínez, 2012; Verdú \& López, 2010).

\section{Bibliografía}

Abbasi, H., \& Mohammadi, S. (2013). The Relationship Between Principals'leadership Styles With Performance Of Physical Education Teachers In Secondary Schools In Western Provinces Of Iran. Asian journal of management sciences \& education, 2(4), 187-193.

Ali, A. Y. S., Sidow, M. A., \& Guleid, H. S. (2013). Leadership styles and job satisfaction: empirical evidence from Mogadishu universities. European Journal of Management Sciences and Economics Vol, 1(1).

Anari, N. N. (2012). Teachers: emotional intelligence, job satisfaction, and organizational commitment. Journal of Workplace Learning, 24(4), 256-269. doi:doi:10.1108/13665621211223379

Arzi, S., \& Farahbod, L. (2014). The impact of leadership style on job satisfaction: A study of Iranian Hotels. Interdisciplinary Journal Of Contemporary Research In Business, 6(3), 171.

Avolio, B. J., Waldman, D. A., \& Yammarino, F. J. (1991). Leading in the 1990s: The four I's of transformational leadership. Journal of European industrial training, 15(4).

Bass, B. (1988). The Inspirational Processes of Leadership. Journal of Management Development, 7(5), 21-31. doi:doi:10.1108/eb051688

Bass, B. M. (1999). Two decades of research and development in transformational leadership. European journal of work and organizational psychology, 8(1), 9-32.

Bass, B. M., \& Avolio, B. J. (1990). Developing transformational leadership: 1992 and beyond. Journal of European industrial training, 14(5).

Bass, B. M., Avolio, B. J., Jung, D. I., \& Berson, Y. (2003). Predicting unit performance by assessing transformational and transactional leadership. Journal of applied psychology, 88(2), 207.

Cardona, P. (2000). Transcendental leadership. Leadership \& Organization Development Journal, 21(4), 201-207.

Chan, S. C. H., \& Mak, W. M. (2014). Transformational leadership, pride in being a follower of the leader and organizational commitment. Leadership \& Organization Development Journal, 35(8), 674-690. doi:doi:10.1108/LODJ-12-09-0076

Chiu, W. Y. B., \& Ng, F. F. (2015). Enhancement of organizational commitment through propensity to trust. Engineering, Construction and Architectural Management, 22(3), 272294. doi:doi:10.1108/ECAM-04-2013-0029

Chong, M. P. M. (2012). Influence behaviors and organizational commitment: a comparative study. Leadership \& Organization Development Journal, 35(1), 54-78. doi:doi:10.1108/LODJ-03-2012-0035 
Cohen, A. (2007). Commitment before and after: An evaluation and reconceptualization of organizational commitment. Human resource management review, 17(3), 336-354.

Dionne, S. D., Yammarino, F. J., Atwater, L. E., \& Spangler, W. D. (2004). Transformational leadership and team performance. Journal of organizational change management, 17(2), 177-193.

Faisal, M. N., \& Al-Esmael, B. A. (2014). Modeling the enablers of organizational commitment. Business Process Management Journal, 20(1), 25-46. doi:doi:10.1108/BPMJ-08-20120086

Farahani, M., Taghadosi, M., \& Behboudi, M. (2011). An exploration of the relationship between transformational leadership and organizational commitment: The moderating effect of emotional intelligence: Case study in Iran. International Business Research, 4(4), p211.

Jain, A. K. (2015). Volunteerism and organisational culture: Relationship to organizational commitment and citizenship behaviors in India. Cross Cultural Management: An International Journal, 22(1), 116-144. doi:doi:10.1108/CCM-11-2013-0167

Jernigan III, I., Beggs, J. M., \& Kohut, G. F. (2002). Dimensions of work satisfaction as predictors of commitment type. Journal of Managerial Psychology, 17(7), 564-579.

Joo, B. K., Yoon, H. J., \& Jeung, C. W. (2012). The effects of core self-evaluations and transformational leadership on organizational commitment. Leadership \& Organization Development Journal, 33(6), 564-582. doi:doi:10.1108/01437731211253028

Komari, N., \& Djafar, F. (2013). Work Ethics, Work Satisfaction and Organizational Commitment at the Sharia Bank, Indonesia. International Business Research, 6(12), p107.

Kuo, Y. K. (2013). Organizational commitment in an intense competition environment. Industrial Management \& Data Systems, 113(1), 39-56. doi:doi:10.1108/02635571311289656

Meyer, J. P., \& Herscovitch, L. (2001). Commitment in the workplace: Toward a general model. Human resource management review, 11(3), 299-326.

Meyer, J. P., Stanley, D. J., Herscovitch, L., \& Topolnytsky, L. (2002). Affective, continuance, and normative commitment to the organization: A meta-analysis of antecedents, correlates, and consequences. Journal of vocational behavior, 61(1), 20-52.

Millward, L. J., \& Hopkins, L. J. (1998). Psychological contracts, organizational and job commitment. Journal of Applied Social Psychology, 28(16), 1530-1556.

Moon, T.-W., Hur, W.-M., Ko, S.-H., Kim, J.-W., \& Yoon, S.-W. (2014). Bridging corporate social responsibility and compassion at work: Relations to organizational justice and affective organizational commitment. Career Development International, 19(1), 49-72. doi:doi:10.1108/CDI-05-2013-0060

Politis, J. D. (2002). Transformational and transactional leadership enabling (disabling) knowledge acquisition of self-managed teams: the consequences for performance. Leadership \& Organization Development Journal, 23(4), 186-197.

Robbins, S., Judge, T. A., Millett, B., \& Boyle, M. (2013). Organizational behaviour: Pearson Higher Education AU.

Rosario-Hernández, E., \& Millán, L. V. R. (2011). La relación entre las manifestaciones de conductas de ciudadanía organizacional con los cinco factores de la personalidad, satisfacción laboral, compromiso organizacional, justicia organizacional y contrato psicológico. Revista Puertorriqueña de Psicología, 22, 72-100.

Rousseau, D. (1995). Psychological contracts in organizations: Understanding written and unwritten agreements: Sage Publications. 
Rousseau, D. M. (1985). Issues of level in organizational research: Multi-level and cross-level perspectives. Research in organizational behavior, 7(1), 1-37.

Rousseau, D. M. (1989). Psychological and implied contracts in organizations. Employee responsibilities and rights journal, 2(2), 121-139.

Rowden, R. W. (2000). The relationship between charismatic leadership behaviors and organizational commitment. Leadership \& Organization Development Journal, 21(1), 3035. doi:doi:10.1108/01437730010310712

Sethuraman, K., \& Suresh, J. (2014). Effective leadership styles. International Business Research, $7(9), \mathrm{p} 165$.

Shepherd, J. L., \& Mathews, B. P. (2000). Employee commitment: academic vs practitioner perspectives. Employee Relations, 22(6), 555-575.

Shim, H. S., Jo, Y., \& Hoover, L. T. (2015). Police transformational leadership and organizational commitment: Mediating role of organizational culture. Policing: An International Journal of Police Strategies \& Management, 38(4), 754-774. doi:doi:10.1108/PIJPSM-05-20150066

Shurbagi, A. M. A. (2014). The Relationship between Transformational Leadership Style Job Satisfaction and the Effect of Organizational Commitment. International Business Research, 7(11), p126.

Silla, J. M. P., Gracia, F. J., Ferreira, L. F., \& Silla, I. (2006). El estado del contrato psicológico y su relación con la salud psicológica de los empleados. Psicothema, 18(2), 256-262.

Solanes Puchol, Á., \& Martínez, Z. (2012). El Estado del contrato psicológico como determinante del compromiso organizacional y la inseguridad laboral percibida: diferencias entre indefinidos y temporales. Revista de Psicología Social Aplicada, 1(1).

Tims, M., Bakker, A. B., \& Xanthopoulou, D. (2011). Do transformational leaders enhance their followers' daily work engagement? The Leadership Quarterly, 22(1), 121-131.

Verdú, F. J. P., \& López, J. R. (2010). Antecedentes del compromiso organizacional: Relaciones con la estabilidad laboral, la autonomía en el puesto y las prácticas de recursos humanos. Estudios financieros. Revista de trabajo y seguridad social: Comentarios, casos prácticos: recursos humanos(323), 239-256.

Vigoda-Gadot, E. (2007). Leadership style, organizational politics, and employees' performance: An empirical examination of two competing models. Personnel Review, 36(5), 661-683.

Wang, X.-H. F., \& Howell, J. M. (2010). Exploring the dual-level effects of transformational leadership on followers. Journal of applied psychology, 95(6), 1134.

Yeh, H. R. (2008). The effects of transformation leadership, organizational culture, job satisfaction on the organizational performance in the non-profit organizations.

Zayas-Ortiz, M., Rosario, E., Marquez, E., \& Gruñeiro, P. C. (2015). Relationship between organizational commitments and organizational citizenship behaviour in a sample of private banking employees. International Journal of Sociology and Social Policy, 35(1/2), 91-106. doi:doi:10.1108/IJSSP-02-2014-0010 